\title{
An insight into anticancer, antioxidant, antimicrobial, antidiabetic and anti-inflammatory effects of quercetin: a review
}

\author{
Muhammad Azeem ${ }^{1,2} \cdot$ Muhammad Hanif $^{1}\left[\right.$ Khalid Mahmood $^{3}$. \\ Nabeela Ameer ${ }^{1}$. Fazal Rahman Sajid Chughtai ${ }^{1}$. Usman Abid ${ }^{1}$
}

Received: 16 September 2021 / Revised: 30 December 2021 / Accepted: 7 January 2022 /

Published online: 30 January 2022

(C) The Author(s), under exclusive licence to Springer-Verlag GmbH Germany, part of Springer Nature 2022

\begin{abstract}
Flavonoids are present naturally in many fruits and vegetables including onions, apples, tea, cabbage, cauliflower, berries and nuts which provide us with quercetin, a powerful natural antioxidant and cytotoxic compound. Due to antioxidant property, many nutraceuticals and cosmeceuticals products contain quercetin as a major ingredient nowadays. Current review enlightened sources and quercetin's role as an antioxidant, antimicrobial, antidiabetic, anticancerous and anti-inflammatory agent in medical field during last 5 to 6 years. Literature search was systematically done using scientific for the published articles of quercetin. A total of 345 articles were reviewed, and it was observed that more than $40 \%$ of articles were about quercetin's use as an antioxidant agent, more than $25 \%$ of studies were about its use as an anticancer agent, and articles on antimicrobial activity were more than $15 \% .10 \%$ of the articles showed anti-inflamamatory effects of quercetin. Literature search also revealed that quercetin alone and its complexes with chitosan, metal ions and polymers possessed good antidiabetic properties. Thus, the review focuses on new therapeutic interventions and drug delivery system of quercetin in medical field for the benefit of mankind.
\end{abstract}

Keywords Quercetin · Antioxidant · Anticancer · Antidiabetic · Antimicrobial · Anti-inflammatory

Muhammad Hanif

Muhammad.hanif@bzu.edu.pk

1 Department of Pharmaceutics, Faculty of Pharmacy, Baha-Uddin-Zakariya University, Multan, Pakistan

2 Hamdard Institute of Pharmaceutical Sciences, Hamdard University, Islamabad Campus, Islamabad, Pakistan

3 Institute of Chemical Sciences, Baha-Uddin-Zakariya University, Multan, Pakistan 


$\begin{array}{ll}\text { Abbreviations } \\ \text { DENV } & \text { Dengue virus } \\ \text { DFT } & \text { Density function theory } \\ \text { DPPH } & \text { 2,2-Diphenyl-1-picrylhydrazyl } \\ \text { DNA } & \text { Deoxy ribonucleic acid } \\ \text { FTIR } & \text { Fourier transformed infrared } \\ \mathrm{H}_{2} \mathrm{O}_{2} & \text { Hydrogen peroxide } \\ \text { MIC } & \text { Minimum inhibitory concentration } \\ \text { MTT } & \text { 3-(4,5-Dimethylthiazol-2-yl)-2,5-diphenyl-2H-tetrazolium bromide } \\ \text { P-gp } & \text { P-glycoprotein } \\ \text { Q/Qu/QCT } & \text { Quercetin } \\ \text { RNA } & \text { Ribonucleic acid } \\ \text { STZ } & \text { Streptozotocin } \\ \text { TEM } & \text { Transmission electron microscopy } \\ \text { Uv } & \text { Ultraviolet } \\ \text { ZOI } & \text { Zone of inhibition }\end{array}$

\section{Introduction}

Polyphenolic molecules possess diverse chemical structures and properties. Flavonoids are present in plants as a member of the polyphenolic class. Recent studies suggested that there are more than 4000 different types of flavonoids present in nature which are divided into sub-classes namely flavonols, flavanones, flavones, catechins, isoflavones, anthocyanidins, dihydroflavonols and chalcones. [1] The class flavonols contain quercetin, a (3,5,7,3',4'-pentahydroxyflavone), present abundantly in vegetables and fruits like apples, onions, berries, red grapes, kales, broccoli, cherries and as well as in tea [2].

Free radicals react quickly with other compounds to capture their electron and gets stable. Attacked molecule loses its electron and itself is converted to a free radical, with the initiation of chain reaction resulting in living cells disruption [3]. From the literature, it was confirmed that hydroxyl groups at 3, 5, 7, 3', 4' of $\mathrm{A}$ and $\mathrm{B}$ rings, the double bond between second and third carbons and fourth carbon's carbonyl group have main role in antioxidant properties of quercetin [1].

The mechanism of action of antibacterial activity of quercetin is thought due to inhibition of $\beta$ ketoacyl carrier protein synthases which are involved in bacterial fatty acid synthesis. Another possible mode of action reported was eradication of the biofilm [4]. Antifungal properties of flavonoids have also been reported. Quercetin exerts its antifungal action with other drugs like fluconazole, amphotericin B, etc., by reducing cell adhesion and affecting genes responsible for biofilm formation [5]. Other antifungal mechanisms of flavonoids include plasma membrane disruption, inhibition of cell wall, cell division, inhibition of efflux system, mitochondrial dysfunction, RNA and protein synthesis inhibition [6].

Wide range of DNA and RNA viruses are sensitive against flavonoids which works by several mechanisms to kill them [7]. Quercetin showed antiviral properties at multiple stages of viral pathogenesis like virus entry, virus replication, 
translation and protein assembly. Literature also confirmed the synergistic use of quercetin with other supplements like vitamin $\mathrm{C}$ to synergize antiviral properties with minimum side effects [8].

Diabetes has become a worldwide major problem whose frequency is increasing day by day, and severe acute and chronic complications are shown by it. Acute complications include hypoglycemia, hyperglycemia and diabetic ketoacidosis which require emergency management, while chronic complications include retinopathy, neuropathy, nephropathy and foot problems [9]. Flavonoid such as quercetin was reported to block enzymes like protein kinases and cyclooxygenases, thus inhibiting apoptosis and cell proliferation. It was also reported that quercetin promotes beta cell regeneration in pancreatic islets, increases insulin release and normalizes blood glucose [10].

Quercetin showed remarkable inhibitory effects on malignant cells like breast, lung, gastric, ovarian, colorectal and hepatic cancers [11]. Anticancer effects of quercetin were reported due to several mechanisms like cell apoptosis, inhibition of angiogenesis, inhibiting P-gp channel, decreasing oncogene expression and regulating specific signaling pathways [12]. In certain diseases like rheumatoid arthritis, ulcer, etc., inflammation worsens the disease profile due to the release of inflammatory mediators. Research confirmed quercetin's anti-inflammatory effect was due to inhibition of proinflammatory cytokines, ATP and nuclear factor kappa B (IkBa) binding sites [13].

Another important feature of quercetin is its chelating property with metals ions like $\mathrm{Fe}^{2+}, \mathrm{Fe}^{3+}, \mathrm{Cu}^{2+}$ and $\mathrm{Ni}^{2+}$. These metal complexes of quercetin had shown antiallergic, antiulcer, antimicrobial, anticancer and anti-Alzheimer's activity [14]. Thus, it is evident that quercetin had shown many advantages and is used in the biomedical field for its pharmacological properties, but some disadvantages like low bioavailability, hydrophobic nature, poor solubility and poor permeability make it difficult for absorption and availability. To overcome this problem, quercetin was complexed with biodegradable polymers more frequently with chitosan to increase its solubility and bioavailability [15].

\section{Sources of quercetin}

Quercetin is found in many fruits, vegetables and in more than twenty species of plants particularly in Mangifera indica, Emblica officinalis, Withania somnifera, Cuscuta reflexa, Santalum album, Curcuma domestica valenton and Foeniculum vulgare. Due to its wide pharmacological activities, quercetin is widely used as a dietary supplement in powder and capsule form -. Several countries reported different dietary intake according to consumption of fruits, vegetables and tea which ranged from 50 to $800 \mathrm{mg} /$ day. Reportedly mean consumption of quercetin in countries like China, Japan, Spain and USA was 18, 16.2, 18.48 and $9.75 \mathrm{mg}$ / day [17]. Table 1 gives the amount of quercetin present in food sources. Figure 1 gives the sources and uses of quercetin. 


\section{Search strategy}

A comprehensive data search was conducted between June and July 2021 on google scholar, ScienceDirect, PubMed, SpringerLink and Wiley online library to include newly published articles to date. Following search terms were used in the search: antioxidant, antimicrobial, anticancer, antidiabetic, anti-inflammatory effects of quercetin, complexes of quercetin with metal ions, and chitosan. There was no limit on sample size, study design or outcome measurement.

\section{Eligibility criteria and data collection}

Articles were considered eligible if they meet the following criteria: (a) in vivo animal study, (b) ex vivo cell line studies, (c) quercetin uses as antioxidant, antidiabetic, anticancer, antimicrobial agents, (d) quercetin conjugates with chitosan, (e) complexes of quercetin with metal ions, (f) use of quercetin in other diseases and (g) Language is English. Studies were excluded if they were patient studies, review articles letters and conference abstracts without details. A total of 345 articles were downloaded and were assessed based on inclusion criteria. After detailed screening, 280 articles were excluded and only 65 research articles were selected for the review.

\section{Properties of quercetin}

\section{Antioxidant property of quercetin}

Quercetin's best-known property to date is its antioxidant profile. It is the most powerful antioxidant flavonoid in nature, protecting our body against free radicals. The antioxidant activity of hesperidin and quercetin was investigated by Srimathi and Vijay against DPPH, nitric oxide, hydroxyl radical, hydrogen peroxide radical, superoxide and reducing power assay. The results revealed that both the compounds

Table 1 Amount of quercetin present in $\mathrm{mg} / 100 \mathrm{~g}$ of food sources

\begin{tabular}{llll}
\hline Food source & $\begin{array}{l}\text { Quercetin in } \\
\mathrm{mg} / 100 \mathrm{~g}\end{array}$ & Food source & $\begin{array}{l}\text { Quercetin in } \\
\mathrm{mg} / 100 \mathrm{~g}\end{array}$ \\
\hline Capers & 233 & Radicchio & 32.0 \\
Onion & 22.0 & Spinach & 27.2 \\
Fennel leaves & 49.0 & Black tea & 2.0 \\
Kale & 23.0 & Red grapes & 1.38 \\
Radish leaves & 70.0 & Broccoli & 2.51 \\
Oregano & 42.0 & Cranberries & 14.0 \\
Dill & 79.0 & Cherries & 2.7 \\
Chili pepper & 32.6 & Red apples & 4.7 \\
Cilantro & 53.0 & Green tea & 2.7 \\
\hline
\end{tabular}




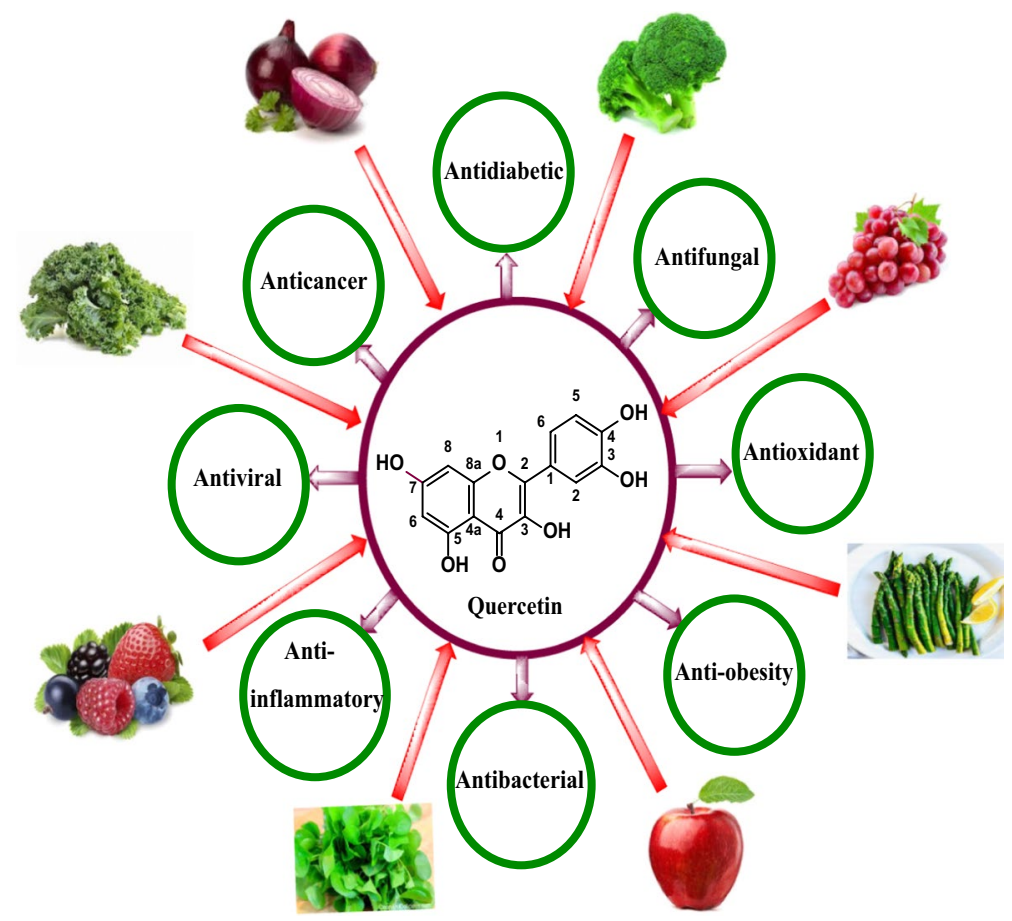

Fig. 1 Sources and uses of quercetin

had greater inhibitory activity against DPPH (IC50 value $41.55 \pm 0.49 \mathrm{mg} / \mathrm{ml}$ and $36.15 \pm 0.30$ ), while ascorbic acid was taken as a reference with (IC50 value $23.2 \pm 0.20 \mathrm{mg} / \mathrm{ml}$ and $31.45 \pm 1.31)$ [3]

Jiao Wang et al. conducted a study in China in which antioxidant activity of quercetin on ovarian aging was reported. This was achieved by giving quercetin at doses of $(12.5,25$ and $50 \mathrm{mg} / \mathrm{kg})$ intragastrical for ninety days, to an in vivo female menopausal rat model. Vaginal smearing was used to determine the Estrous cycle. In vitro tests were also conducted by taking rat ovarian granulosa cell-cultured treated with $400 \mu \mathrm{M}$ of $\mathrm{H}_{2} \mathrm{O}_{2}$ alone and 5, 20 and $50 \mu \mathrm{M}$ doses of $\mathrm{H}_{2} \mathrm{O}_{2}$ plus quercetin. Radioimmunoassay was used to determine levels of luteinizing, follicle-stimulating, progesterone and estradiol hormones. Western blot test was used to detect the level of expression of oxidative stress-related genes, catalase and glutathione synthetase in ovarian cells. The results of in vivo study showed no impact of quercetin on ovarian morphology, hormone secretion and estrous cycle in rat models. However, levels of oxidative stress-related genes were increased at a low dose of quercetin. In vitro assay revealed diminish in cell viability by $\mathrm{H}_{2} \mathrm{O}_{2}$-initiated oxidative stretch [18].

In various neurodegenerative diseases, major reactive oxygen species is hydrogen peroxide. A study was conducted in Japan to investigate the neuroprotective part 
of quercetin against $\mathrm{H}_{2} \mathrm{O}_{2}$ actuated apoptosis in human neuronal SH-SY5Y cells. Reduction in lactate dehydrogenase and $\mathrm{H}_{2} \mathrm{O}_{2}$-mediated cytotoxicity was observed due to quercetin. Additionally, pro-apoptotic Bax gene level was suppressed and antiapoptotic gene Bcl-2 gene level was enhanced by quercetin in neuronal cells. Thus, quercetin acts as preventive neurodegenerative maladies caused by oxidative push and apoptosis [19].

Marija Lesjak et al. reported antioxidant activity of six derivatives of quercetin, namely tamarixetin, isorhamnetin, quercetin-3-O-glucuronide, isorhamnetin-3-Oglucuronide, 3,4'-diOglucoside, quercetin-3,5,7,3'4'-pentamethylether compared with onion extract and standards butylated hydroxytoluene (BHT) and aspirin. In vitro DPPH assay was used to determine the antioxidant profile of all samples which showed that quercetin, tamarixetin, isorhamnetin and quercetin-3-O-glucuronide showed eminent antioxidant movement, essentially higher than BHT. Quercetin aglycone appeared most strong antioxidant property, but the derivatization of its hydroxyl bunches altogether diminished the antioxidant movement of its subsidiaries. It was concluded that antioxidant potential was straightforwardly corresponding to the number of free hydroxyl bunches in test samples [20].

Yan-Zhen Zheng et al. also confirmed the role of hydroxyl groups of quercetin for producing an antioxidant profile. They conducted a theoretical study on propolis in which flavonoids contribute greatly among the multiple components. Density functional theory (DFT) calculation was used with different mechanisms namely, sequential proton loss transfer (SET-PT), hydrogen atom transfer (HAT) and sequential proton loss transfer (SPLET) done in their study to assess antioxidant properties of quercetin and its glucosides in gas and fluid stage (ethanol, water). Antioxidative sequence in the gas phase was: quercetin $>$ quercetin-5-O-glucoside $>$ quercetin-7-Oglucoside $>$ quercetin-3-O-glucoside $>$ quercetin-3'-O-glucoside $>$ quercetin-4'-Oglucoside. While sequence in solvent phases was: the sequence is similar with the proton affinity values: quercetin-4'-O-glucoside $>$ quercetin-5-O-glucoside $>$ quercetin $>$ quercetin3-O-glucoside $>$ quercetin-7-O-glucoside $>$ quercetin-3'-O-glucoside. It was affirmed that $\mathrm{OH}$ groups in B-ring and $\mathrm{C}$-ring contribute primarily to the antioxidative exercises of quercetin and glucosides compared with A-ring [21]. Table 2 gives a summary of antioxidant, antimicrobial, antidiabetic, anticancer and antiinflammatory effects of quercetin reported in this review.

\section{Antimicrobial property of quercetin}

\section{Antibacterial property}

Vikas Pahal et al. reported an antibacterial profile of quercetin extracted from $C$. officinalis. In vitro studies were performed against $S$. aureus using different organic extracts, and an in silico study was done on four important bacterial enzymes. namely peptide deformylase, gama hemolysins, DNA primases and undecaprenyl pyrophosphate synthase using AutoDock Vina 1. Results for in vitro study revealed the activity of methanolic extract at $20 \mathrm{mg} / \mathrm{ml}$ of quercetin completely inhibits the growth of $S$. aureus. In silico results revealed that quercetin was found to be the as it 
were auxiliary metabolite that hindered three proteins of $S$. aureus (DNA primases, undecaprenyl pyrophosphate synthase and peptide deformylase) [22].

Sergio Dias da Costa Junior et al. reported antibiofilm and antibacterial properties of quercetin against resistant $S$. aureus and $S$. saprophyticus. Microdilution method was used for determining MIC values. It was concluded that quercetin showed antibacterial activity against methicillin-susceptible $S$. aureus (MSSA) (MIC $=250 \mu \mathrm{g}$ / $\mathrm{ml}$ ), methicillin-resistant $S$. aureus (MRSA) (MIC $=500 \mu \mathrm{g} / \mathrm{ml})$, vancomycin-intermediate $S$. aureus (VISA) (MIC $=125$ and $150 \mu \mathrm{g} / \mathrm{ml}$ ), S. saprophyticus resistant to oxacillin (MIC $=62.5$ to $125 \mu \mathrm{g} / \mathrm{ml}$ ), vancomycin-resistant $S$. aureus (VRSA) and $S$. saprophyticus resistant to oxacillin and vancomycin (MIC $=500$ to $1000 \mu \mathrm{g} / \mathrm{ml}$ ). $46.5 \pm 2.7 \%$ and $39.4 \pm 4.3 \%$ of the $S$. aureus biofilm were inhibited by quercetin at $\mathrm{MIC} / 2$ and MIC/4, respectively, while that of S. saprophyticus biofilm at $51.7 \pm 5.5 \%$ and $46.9 \pm 5.5 \%$. Thus, it was confirmed that quercetin acts as a great antibacterial agent naturally [23].

Artur Adamczak et al. reported the antibacterial profile of 13 flavonoids, namely apigenin, chrysin, flavones, isoorientin, isovitexin, kaempferol, luteolin, naringin, orientin, quercetin, rutin, vitexin and vitexin 2-o-rhamnoside along with 6 organic acids namely rosmarinic acid, quinic, malic, citric chlorogenic and salicylic acid. MIC values of all the test compounds were determined by microdilution method using four clinical isolates, i.e., Gram-positive ones: E. faecalis and S. aureus, and Gram-negative bacteria: $E$. coli and $P$. aeruginosa. It was concluded that all tried compounds gave antimicrobial properties to a greater extent against Gram-negative bacteria as compared to Gram-positive bacteria. The study also confirmed the presence of hydroxyl groups in phenyl rings did not have any influence on their activity level. However, a significant increase in activity of the hydroxy derivatives of flavone was observed against $S$. aureus [24].

Feng Lia et al. reported a novel complex consisting of chitosan-based nanoparticles consisting of catechin and quercetin. Ionic gelation method was used to synthesize his complex by reacting chitosan and sodium tripolyphosphate using genipin. The objective was to enhance the antibacterial and antioxidant profile of catechin and quercetin. The average particle size obtained was $180.4 \mathrm{~nm}$ with a zeta potential of $31.79 \pm 1.28 \mathrm{mV}$. In in vitro, drug release showed sustained release of drug from the nanoparticles. Higher scavenging property of the nanoparticles was reported on ABTS, DPPH, $\mathrm{OH}$ and $\mathrm{O}_{2}$ radicals. Antibacterial assay (minimum inhibitory concentration and zone of inhibition) of pure compounds and blank and drug-loaded nanoparticles was done on three bacterial strains, i.e., S. aureus, B. subtilis and E. coli. The results confirmed the enhanced antibacterial profile of nanoparticles made of chitosan (G-C-Q NPs), loaded with quercetin and catechin. Thus polymeric particles are a good choice as drug delivery system for bacterial infections [25].

Sarangapani Sreelatha and Jayachitra conducted a very interesting study in India and used a fungal Strain of Aspergillus niger for the conversion of rutin to quercetin with enhanced production rate and minimum toxic wastes. The produced quercetin was evaluated for various effects including antibacterial effects against $S$. aureus, $E$. coli and P. aeruginosa. The release of ROS from quercetin at the surface of biofilms of the three bacterial strains results in biofilm disruption and cell death of microbes. A greater zone of inhibition of quercetin was observed against $S$. aureus. 


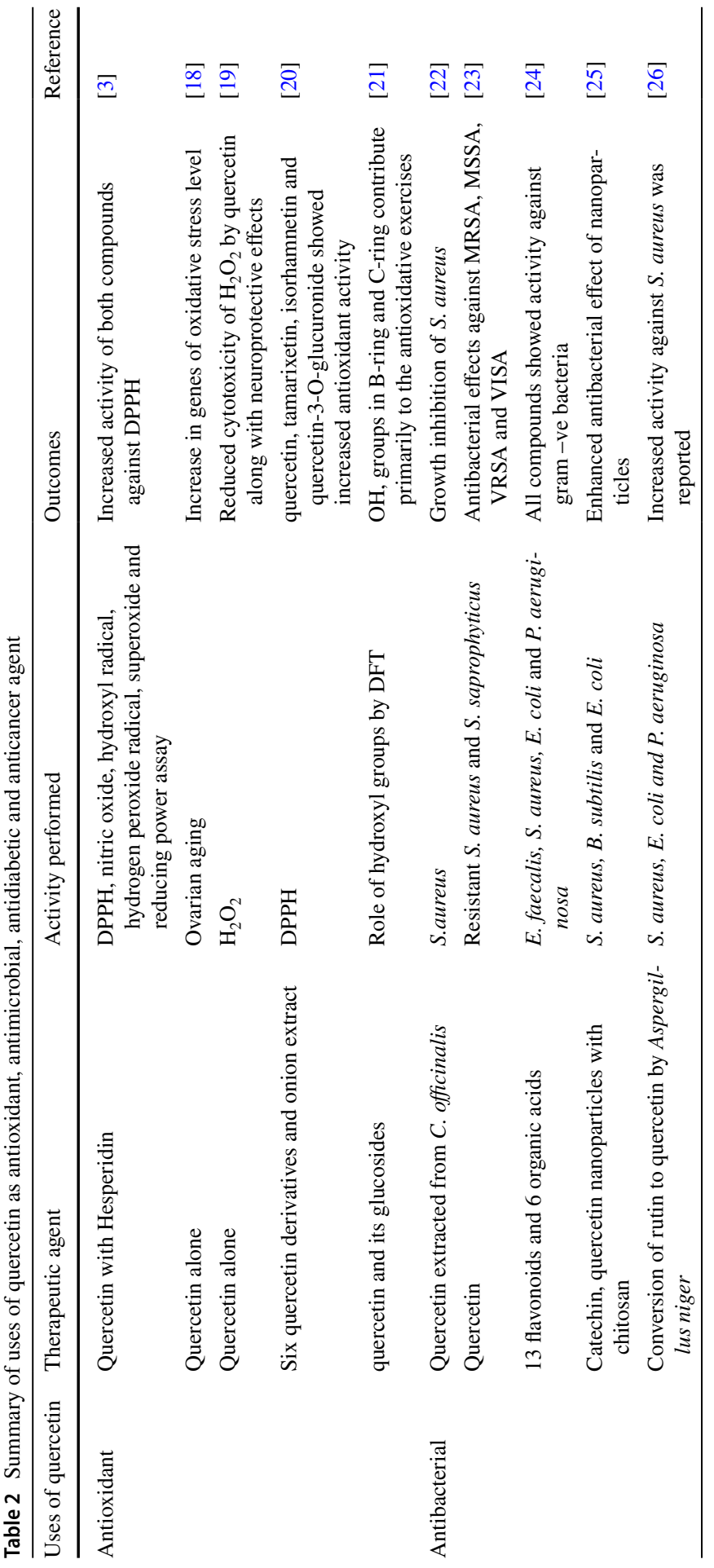




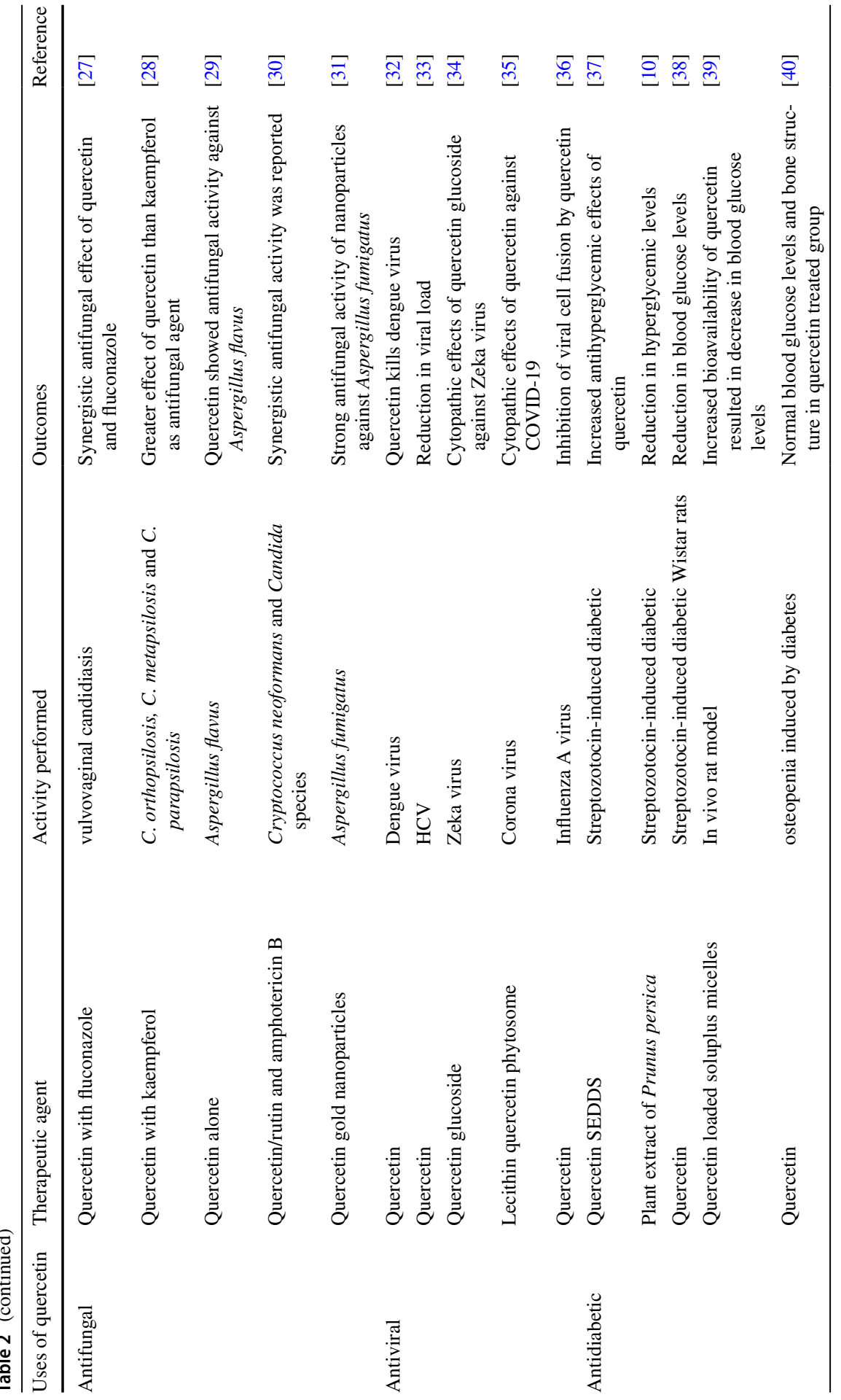




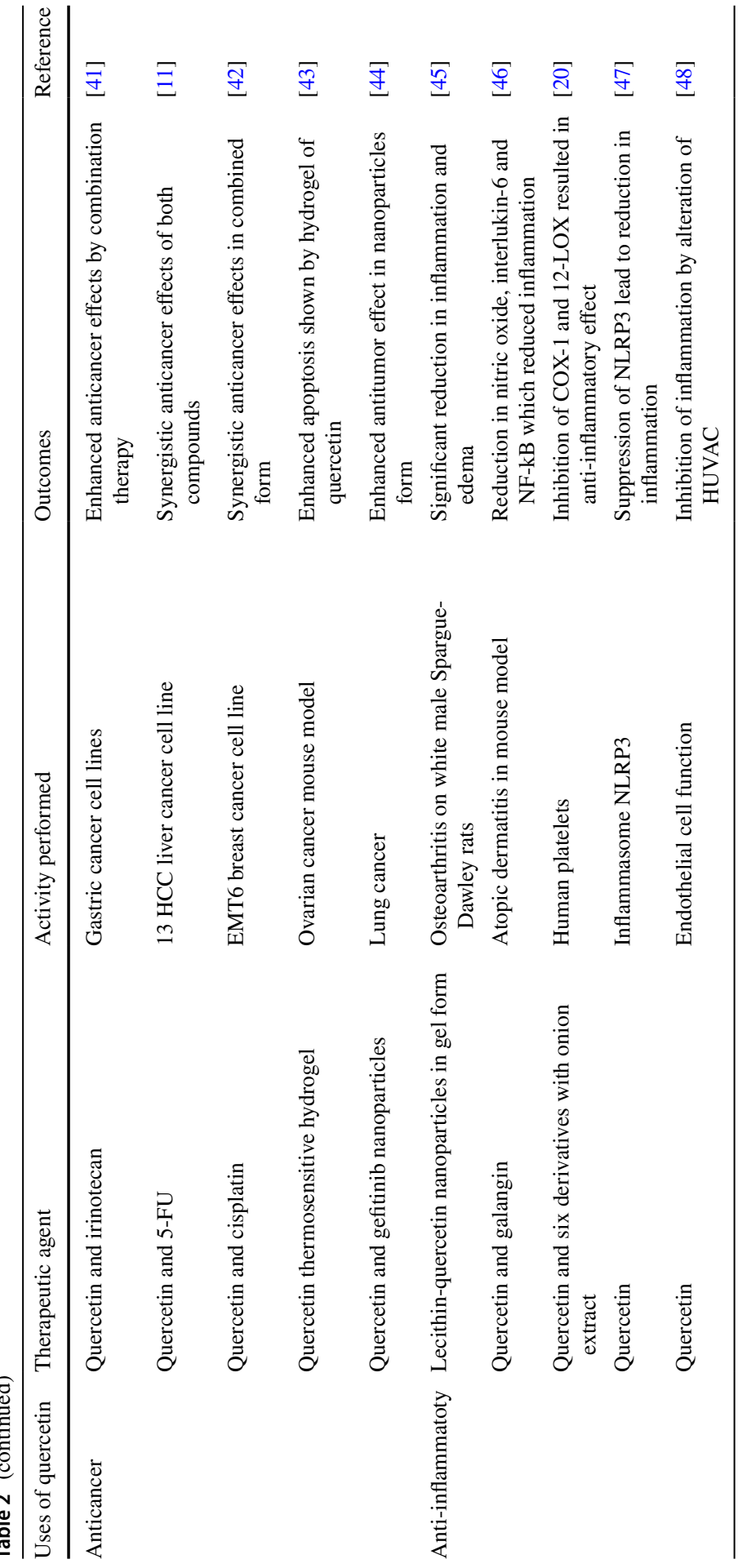


Thus, this study gave new insights into the biotransformation of rutin into quercetin to achieve a rich dietary supplement for better antimicrobial effects [26].

\section{Anti-fungal property}

Mei Gao et al. in a study reported the management of vulvovaginal candidiasis (VVC) with quercetin (QCT)-assisted fluconazole (FCZ). Fifteen clinical isolates of C. Albicans were isolated from patients suffering from VVC. The study confirmed the synergistic effect of QCT with FCZ at doses $64 \mathrm{ug} / \mathrm{ml}$ and $128 \mathrm{ug} / \mathrm{ml}$ to inhibit antifungal biofilms. The fungal burden was reduced in vaginal mucosa in murine VVC by treating with $5 \mathrm{mg} / \mathrm{kg}$ of QCT and $20 \mathrm{mg} / \mathrm{kg}$ of [27].

Another study was conducted by Marcos Fabio Gadelha Rocha et al. in which MIC of kaempferol and quercetin was assessed against $C$. orthopsilosis, C. metapsilosis and C.parapsilosis strains. It was analyzed that kaempferol and quercetin decreased the metabolic activity and biomass of all the fungal strains. The MIC of quercetin ranged from 0.5 to $16 \mathrm{ug} / \mathrm{ml}$, while that of kaempferol was $32-128 \mathrm{ug} / \mathrm{ml}$. Thus these compounds can be used as antifungals [28].

In India, a study was conducted by Sonia Shishodia et al. which reported the survival techniques of Aspergillus flavus during germination process. The study was aimed to observe quercetin effect on signaling pathways of Aspergillus flavus during germination using SEM. At 4- and 7-h intervals, a significant rise in heat shock proteins and calcium signaling pathways were observed by real-time quantitative PCR in response to quercetin. Higher levels of calcium kinase, cAMP, Rhogdp, Plc and Pkc encoding genes were observed in groups treated with quercetin. Thus, the data suggested the antifungal potential of quercetin [29].

Amphotericin B is the choice of drug in most of systemic fungal infections, particularly cryptococcosis. But due to its side effects, its use is limited. Amphotericin $\mathrm{B}$ and quercetin/rutin were analyzed for their antifungal activity alone and in combinations on Cryptococcus neoformans and Candida species. MIC value of amohotericin B was significantly reduced when used with quercetin and rutin on C. neoformans both on clinical isolates and on ATCC strain. The study concluded less use of amphotericin B to prevent its cytotoxic effects and to enhance antifungal activity when used with quercetin and rutin [30].

Felipe Guzansky Milanezi et al. reported quercetin capped gold nanoparticles (AuNPsQct) having antioxidant, antimicrobial and cytotoxic activity. They were prepared by using trichlorogold-hydrochloric acid, quercetin and aqueous sodium citrate solution. Confirmation of nanoparticles was done by FTIR, Uv and TEM analysis. (AuNPsQct) nanoparticles were evaluated for antifungal activity along with antibacterial and antioxidant activity. Results of antifungal activity revealed strong antifungal activity with concentrations from 0.1 to $0.5 \mathrm{mg} / \mathrm{mL}$ was observed for Aspergillus fumigatus [31].

\section{Antiviral property}

Dengue virus infection (DENV) is still a problem in countries like Indonesia, due to no specific antiviral medicine available for its management. Quercetin, a 
plant-derived flavonoid, was investigated for its antiviral activity, against DENV. The study was designed in human cell line Huh 7 it-1 infected with DENV strain-2 New guinea C. MTT assay was performed, and IC50 and CC50 for quercetin were found to be $18.41 \mu \mathrm{g} / \mathrm{mL}$ and $217.113 \mu \mathrm{g} / \mathrm{mL}$, respectively. The results confirmed quercetin as an effective antiviral agent against DENV-2 [32].

Anti-hepatitis C (HCV) virus activity of quercetin was investigated by Angela Rojas et al. in Spain. At different steps of the HCV life cycle in Huh-7.5 cells and primary hepatocytes $(\mathrm{PHH})$ infected with $\mathrm{HCVcc}$, quercetin antiviral effects were observed. Significant viral load reduction was observed in both cell types up to $85 \%$ in Huh7.5 and 92\% in PHH. A 65\% reduction in infectivity was observed when quercetin was directly applied to viral particles. Interestingly quercetin also prevented the up-regulation of diacylglycerol acyltransferase (DGAT) and localization of the HCV center protein to the surface of lipid beads. Thus, it was concluded that quercetin's antiviral effects were host-mediated against HCV [33].

A quercetin derivative containing a glucoside molecule was tested for its in vitro and in vivo antiviral activity against Zeka viral strains. In vitro test of quercetin3- $\beta$-O-D-glucoside (Q3G) was performed on Vero cells with PLCal-ZV strain. Serial dilutions ranging from $0.67 \mu \mathrm{mol} / \mathrm{L}$ to $10.76 \mu \mathrm{mol} / \mathrm{L}$ were applied on the infected Vero cells, and after 2 and 4 days virus RNA was extracted and analyzed by real-time qPCR. EC50 and EC90 results revealed cytopathic effects of Q3G at $1.2-1.3 \mu \mathrm{mol} / \mathrm{L}$ and $1.5 \mu \mathrm{mol} / \mathrm{L}$, respectively. The in vivo assay was performed on immunocompromised mice lacking the receptor for type I interferon treated with PRVABC59 strain. Zika virus strain and Q3G were administered at dose $50 \mathrm{mg} /$ $\mathrm{kg}$ up to 7 days intraperitoneally. The results revealed better survival rates and less weight loss in animals given Q3G [34].

Based on the antiviral potential of quercetin, a new pharmaceutical dosage form consisting of quercetin and lecithin quercetin phytosome (QP) was made in Italy and tested on SARS-CoV-2 patients. An open-labeled randomized controlled clinical study was conducted for two weeks. 42 COVID-19 patients were enrolled; among them, 21 were treated with standard care (SC); remaining 21 were treated with QP. Results after one week showed a complete negative profile in 16 patients in the QP group, with 12 patients showing all diminished symptoms. In the SC group, 17 patients took more than two weeks to give a negative test of COVID-19, one tested negative at 3rd week and one patient was positive till expired at day 20. Hence, it was concluded that QP abbreviates the time of test change from positive to negative at the side diminishment in indications and seriousness of COVID-19 [35].

Regular pandemics and scourges with tall mortality and dismalness were caused by flu A infections (IAVs). During infection, glycoprotein hemagglutinin plays a vital role, making it a potential target for anti-influenza drugs. Different strains were tested for antiviral activity of quercetin including A/Puerto Rico/8/34 H1N1, A/Aichi/2/68 H3N2 and A/FM-1/47/1 H1N1. IC50 values of quercetin against these viral strains were $7.756 \pm 1.097,6.225 \pm 0.467$ and $2.738 \pm 1.931 \mu \mathrm{g} / \mathrm{mL}$. The study revealed the interaction of quercetin with hemagglutinin subunit and inhibited viral cell fusion. Thus a natural source with favorable benefits can be used as an antiviral agent [36]. Figure 2 depicts the role of quercetin as an anticancer, antidiabetic, antioxidant and antimicrobial agent. 


\section{Antidiabetic property of quercetin}

Diabetes mellitus (DM) has emerged as a major health problem in Saudi Arabia due to change in lifestyle of the population. Saudi Arabia ranked seventh in the world and second in the Middle East in the occurrence of DM according to the World Health Organization. For controlling diabetes, quercetin was selected and a self-emulsifying drug delivery system (SEDDS) was prepared. The streptozotocininduced diabetic rat model was used for studying the antidiabetic effect of quercetin as compared to reference drug glibenclamide. Results confirmed the blood sugar level lowering capacity of quercetin which was due to insulin release from beta cells [37].

A plant named Prunus persica was evaluated for its antidiabetic activity in India along with antioxidant and anti-adipogenic effects. Leaf's extract of ethyl acetate was taken, and at doses 100 and $200 \mathrm{mg} / \mathrm{kg}$ per oral was given to streptozotocin (STZ)-induced diabetic rat models. Significant reductions in hyperglycemic levels were reported at $200 \mathrm{mg} / \mathrm{kg}$ dose along with improvement in body weight and lipid profile. The extract also showed an antioxidant profile confirming the role of quercetin in the management of blood glucose and lipid levels in diabetes mellitus [10].

Oxidative stress due to hyperglycemia is a common complication associated with diabetes. Mina Hemmati et al. investigated two enzymes expression in glucose metabolism, namely glucokinase and glucose-6-phosphatase. For this, twenty-four adult Wistar rats were divided into three groups namely, control, streptozotocininduced diabetic rats and quercetin-treated group $(15 \mathrm{mg} / \mathrm{kg}$, intraperitoneally). At the end of the 21 day treatment period, a significant reduction in blood glucose and malondialdehyde levels were shown by quercetin. The mRNA levels of HSP70, HSP27, HSF-1 and glucose 6 phosphatase decreased significantly, while glucokinase expression increased in response to quercetin $(p<0.05)$. These findings are beneficial to health care professionals for using quercetin as a nutritional supplement [38].

Therapeutic applications of quercetin are hampered by its low solubility in gastrointestinal fluids. To enhance its solubility profile for the assessment of antidiabetic effects, Juhi Singh et al. used the co-solvent evaporation method to formulate quercetin-loaded Soluplus micelles. The micelles formed were characterized and assessed for in vivo and in vitro studies. The size of the micelles was less than $100 \mathrm{~nm}$, while entrapment efficiency was greater than $70 \%$. In vitro results showed a $28.75 \%$ release of quercetin up to $24 \mathrm{~h}$. In vivo results revealed significantly lower blood glucose levels in the group treated with quercetin-loaded Soluplus micelles. Bioavailability profile improved to $1676 \%$ as compared to a pure drug suspension. Thus, it was concluded that by making micelles of quercetin, enhanced antidiabetic effects were reported due to the increased bioavailability of quercetin [39].

The sixth classical complication of diabetes mellitus is diabetic osteopenia, caused by high blood glucose which triggers oxidative stress. For investigating quercetin's effect on osteopenia induced by diabetes, Mariam A. Abu Ayana et al. used 24 adult male rats which were divided into three groups, namely control, diabetic-induced and quercetin-treated $100 \mathrm{mg} / \mathrm{kg} /$ day. Streptozotocin was used to induce diabetes. All the rats were killed after 12 weeks; mandibles were dissected 


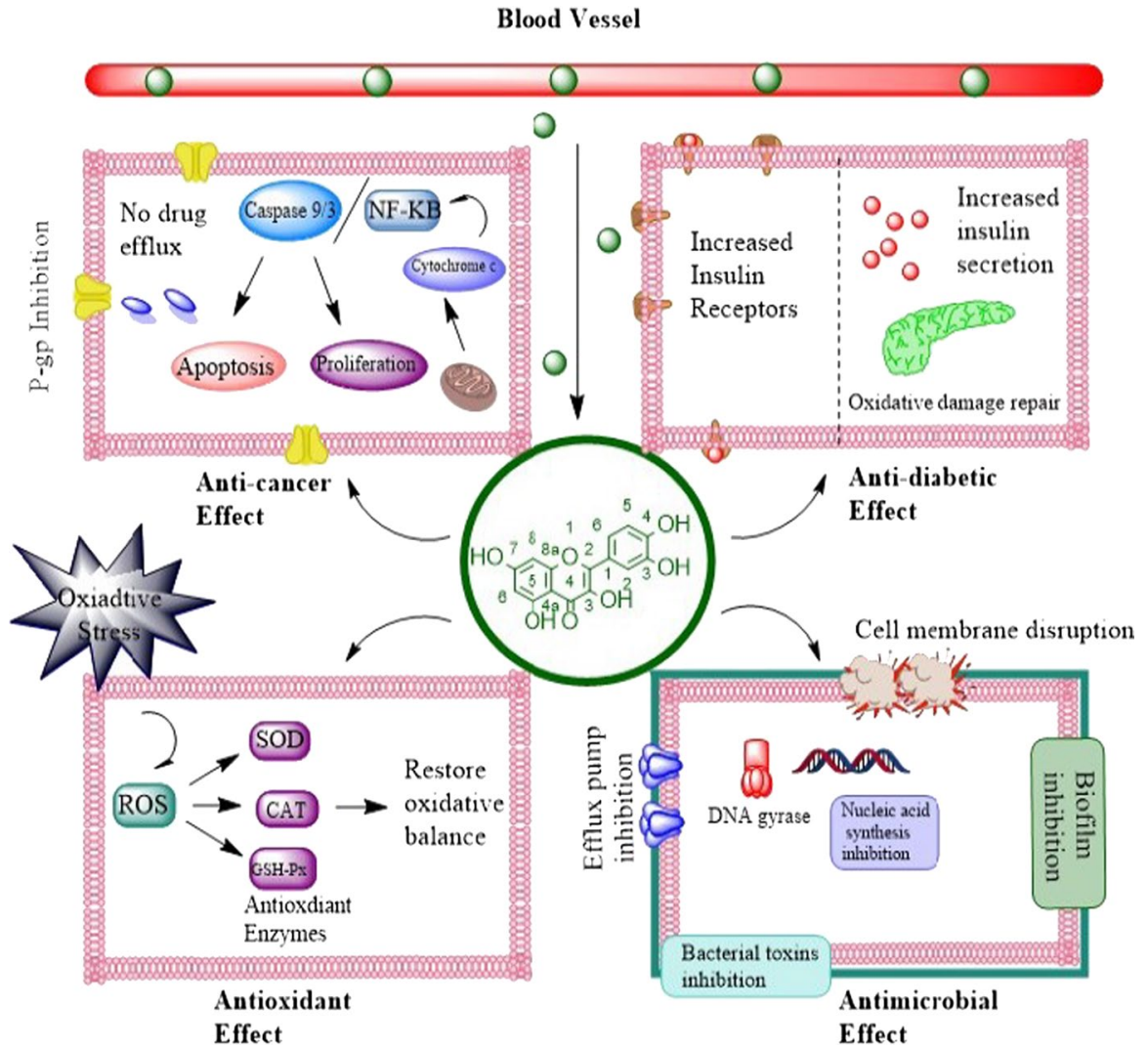

Fig. 2 Cellular mechanism of quercetin as anticancer, antidiabetic, antioxidant and antimicrobial agent

and prepared for scanning electron microscopy (SEM) and energy-dispersive X-ray microanalysis. Results showed increase blood glucose level with an irregular bone surface in the diabetic group, while both blood glucose level and alveolar bone surface returned to normal in quercetin-treated group. This confirms the protective effect of quercetin in restoring bone architecture induced due to diabetes [40].

\section{Anticancer property of quercetin}

Several studies confirmed the ability of quercetin to potentiate the efficacy of anticancer drugs. A study was conducted in Taiwan, to study the synergistic effect of quercetin, irinotecan and its metabolite SN-38 in the gastric cancer cell line of humans both in vitro and in vivo. Results for in vitro analysis revealed that lowdose SN-38 combined with quercetin showed comparable therapeutic effects as that of high-dose $\mathrm{SN}$-38. $\beta$-catenin protein expression level increased in the group managed with high-dose SN-38, while it was less in quercetin alone and as well in combined low doses of $\mathrm{SN}-38$ with quercetin. In vivo results showed high levels 
of cyclooxygenase- 2 and several markers of epithelial-mesenchymal such as Twist1 and ITG $\beta 6$ in rat models treated with irinotecan, while their levels were low in group treated with quercetin combined with a low dose of irinotecan. The results confirmed the enhanced efficacy of quercetin with irinotecan/SN-38 in the human AGS cell line [41].

Several studies showed anticancer effects of quercetin by apoptosis and suppress cell proliferation of breast, lung, oral and prostate cancer. Toru Hisaka et al. for the first time examined antitumor effects of quercetin on thirteen liver cancer cell lines $13 \mathrm{HCC}$ in vitro. Quercetin alone and in combination with 5-fluorouracil (5-FU) was given, and cell viability was performed by MTT assay. The results showed synergistic activity of quercetin and 5-FU by cycle arrest via induction of apoptosis [11].

Quercetin's anticancer effect along with cisplatin was observed in a study conducted in Korea. EMT6 a breast cancer cell line was used to induce tumor in mice and was subcutaneously injected. Animals were divided into four groups namely, control (C), quercetin (Q), cisplatin (CP) and cisplatin + quercetin (CP+Q). Results revealed the enhanced anticancer profile of $(\mathrm{CP}+\mathrm{Q})$ group as compared to other groups. Serum creatinine and BUN levels were less, and alkaline phosphatase and renal $\gamma$-glutamyl transpeptidase activities were significantly higher in $(C P+Q)$ group. These findings suggest synergistic activity of quercetin and cisplatin on breast cancer cell lines along with decreased side effects of cisplatin in animal models [42].

Among the most prevalent cancers in females, ovarian cancer ranks seventh worldwide. It is a serious malignancy affecting the reproductive system in females. Guangya $\mathrm{Xu}$ et al. enhanced the effect of quercetin as an anticancer agent but improving its solubility profile. For this, a quercetin-loaded thermo-sensitive injectable hydrogel system (Qu-M-hydrogel) was made based on nanotechnology. Quercetin was encapsulated using MPEG-PCL and later added into a thermosensitive hydrogel. The formulated hydrogel system showed a slower release of quercetin in vivo. Qu-M-hydrogel composites showed enhanced apoptosis and inhibition of cell growth effects on the ovarian cancer mouse model (SKOV-3) [43].

Investigating the role of quercetin as an anticancer agent against lung cancer, researchers in China conducted a study in which nanoparticles were made comprised of gefitinib and quercetin. Antitumor activity was conducted both in vitro and in vivo. Nanoparticles formed showed good entrapment and release of both drugs up to $12 \mathrm{~h}$. Results for both in vitro and in vivo studies confirmed the enhanced antitumor effect of gefitinib and quercetin [44].

\section{Anti-inflammatory property of quercetin}

Deantari karliana et al. formulated nanoparticles gel consisting of lecithin-quercetin injected into chitosan-tocopherol polyethylene glycol succinate (TPGS) to study the effects on osteoarthritis. White male Sprague-Dawley rats (2-3 month-old) weighed 200-300 g were used for this study and divided into five groups. Osteoarthritis was induced by giving $3 \mathrm{mg} / 0.05 \mathrm{ml}$ mono-iodoacetate in $0.9 \%$ saline. The amount of quercetin administered through nanoparticle gel was $0.84,1.68$ and $3.37 \mathrm{mg} / \mathrm{g}$ gel. 
Significant reduction in inflammation and edema was observed at dose $3.37 \mathrm{mg} / \mathrm{g}$ confirming the role of quercetin in cartilage prevention and reduction in inflammation [45].

A study reported in Korea gave the anti-inflammatory mechanism of two flavanols, namely galangin and quercetin in lipopolysaccharide-stimulated RAW264.7 macrophages. Atopic dermatitis induced by a 2,4-dinitrochlorobenzene mouse model was used. Both the compounds were given alone and also in combination with study the synergistic effect. Results of the study revealed that both these compounds reduce nitric oxide production, interleukin-6 and nuclear factor NF-kB. From histological and ear thickness measurement, it was concluded that reduction in inflammation and $\operatorname{IgE}$ levels were greatly reduced when these flavonoids were used in combinations. Thus, quercetin and galangin combination provided new ways for the prevention of $\mathrm{AD}$ [46].

Marija Lesjak et al. reported anti-inflammatory activity of six quercetin derivatives, namely (tamarexitin, quercetin-3-O-glucuronide, isorhamnetin, isorhamnetin-3-o-glucoside, quercetin-3,4-di-oglucoside, quercetin-3,5,7,3',4'-pentamethyl ether) as compared to onion extract. Ex vivo COX-1 and 12-lipoxygenase (12-LOX) assays using human platelets were applied for assessing anti-inflammatory potential. The method was based on the inhibitory potential of compounds like eicosanoids and prostaglandins catalyzed by inflammatory enzymes response, COX-1 and 12-LOX. Results revealed noticeable anti-inflammatory activity of quercetin and its derivative in the following order: tamarixetin $>$ quercetin = quercetin-3,4'-di-Oglucoside $>$ isorhamnetin $=$ quercetin-3-O-glucuronid $>$ isorhamnetin-3-O-glucoside $>$ quercetin-3,5,7,3', $4^{\prime}$-penthamethylether [20].

Severe inflammation and inflammatory pneumonia is the risk factor associated with SARS-Cov-2. Cytokine storm is responsible for death in infected patients mainly due to acute lung injury, acute respiratory distress syndrome and multiple organ dysfunction syndromes. The study revealed that NLRP3 is an inflammasome responsible for the activation of several inflammatory mediators like NRF2, SIRT1 and TXNIP. Quercetin by affecting this inflammasome successfully suppressed NLRP3 and thus acts as a potential treatment for severe inflammation and in lifethreatening conditions like COVID-19 [47].

Endothelial cell function is affected by cytokines and pro-inflammatory stimuli such as high blood glucose levels. Quercetin was investigated to reduce the harmful effects of hyperglycemia and inflammatory conditions on vascular endothelium. The metabolomics approach was used to identify and quantify 27 human umbilical veins endothelial cell (HUVEC) metabolites. A significant increase in lactate and glutamate concentrations was observed with the treatment of high glucose concentrations in HUVECs. Lactate and adenosine triphosphate concentrations were inhibited by quercetin, while inosine levels were increased. TNF- $\alpha$ and quercetin reduced pyruvate concentration. Quercetin altered HUVEC metabolites balance toward less inflamed phenotype, both alone and in the presence of pro-inflammatory stimuli [48]. 


\section{Complexes of quercetin with metal ions}

Mousumi Patra et al. reported a novel complex of calcium phosphate-quercetin-nanoparticles (CPQN) which was synthesized by precipitation method. For this, quercetin, calcium nitrate and ammonium hydrogen phosphate were used as precursors, and stabilization was done by sodium citrate. Nanoparticles formed had a unique property of color change at different $\mathrm{pH}$ which could be used as a $\mathrm{pH}$ indicator. These nanoparticles were used as fluorophores to mark biological cells. Besides this, $\mathrm{CPQN}$ was investigated for antioxidant activity on mouse neuroblastoma cell N2A, by $\mathrm{H}_{2} \mathrm{O}_{2}$-induced oxidative stress. The nanoparticles exhibited remarked antioxidant properties and thus emerged as a unique beneficial moiety [49].

Hassanien et al. formulated a new complex for diabetes consisting of cobalt complexed with quercetin, cobalt-quercetin complex (CQC) by mixing quercetin solution and cobalt chloride solution in 1:2 molar concentrations. Swiss albino adult male rats weighing 130-165 g were taken for the study, and diabetes was induced by streptozotocin $50 \mathrm{mg} / \mathrm{kg}$. Animals were divided into four groups, and a dose of $\mathrm{mg} \mathrm{Co} 2+/ \mathrm{kg}$ was given for 15 days. Several histopathological and biochemical parameters were assessed along with blood glucose levels, and it was found that CQC efficiently reversed diabetes-induced changes through its strong antioxidant activity. Antihyperglycemic effects of CQC were comparable with insulin [50]. Another study was conducted in Egypt to investigate metal complexed with quercetin for the management of diabetes. Here, $\mathrm{Zn}$ (NO3)2.6H2O was added in the presence of ammonia solution to formulate quercetin-zinc complex having formula $\mathrm{ZnQNO} \mathrm{H}_{3} \mathrm{H}_{2} \mathrm{O} \cdot 5 \mathrm{H}_{2} \mathrm{O}$ which was confirmed by ${ }^{1} \mathrm{HNMR}$. For analyzing the antidiabetic profile of the complex formed, a streptozotocin (STZ)-induced diabetes rat model was used. Seventy male albino rats were divided into seven different groups, namely control, diabetic untreated group and diabetic groups treated with either bone marrow-derived mesenchymal stem cells (MSCs) and/or Q and/or Q/Zn or their combination. Several biochemical parameters along with glycosylated hemoglobin were analyzed. The findings revealed improvement in pancreatic and pulmonary function of combined therapy of Q/Zn and MScs for the treatment, hyperglycemia and oxidative injury induced by diabetes mellitus [51].

Quercetin possesses permeability glycoprotein (P-gp) inhibitory activity which could be enhanced by conjugating it with metals. Six different metal complexes of quercetin $(\mathrm{Cu}, \mathrm{Zn}, \mathrm{Co}, \mathrm{Vd}, \mathrm{Mo}, \mathrm{Ni})$ were synthesized and analyzed in vitro by everted sac intestinal model of rats. The permeability of atorvastatin was observed in different control and experimental groups. Drug content was analyzed by HPLC using mobile phase water and acetonitrile (40: $60 \mathrm{v} / \mathrm{v})$ adjusted at $\mathrm{pH} 2.8$ using phosphate buffer. Quercetin complexes of $\mathrm{Cu} / \mathrm{Co} / \mathrm{Ni}$ decreased the permeability of atorvastatin significantly as compared to other metal complexes of quercetin and can be used as p-gp inhibitors [14]. 


\section{Complexes of quercetin with chitosan}

Yuzhi $\mathrm{Mu}$ et al. reported a novel $\mathrm{pH}$ responsive nano-micelle based on quercetin, chitaconic anhydride and chitosan (QT-CA-CS). Anticancer drug doxorubicin was encapsulated in QT-CA-CS self-assembled nano-micelles by ultrasound method. The advantage of this complex was that drug efflux from the cancerous cell was inhibited due to inhibition of the P-glycoprotein pump. At an acidic $\mathrm{pH}$ of 4.5, greater drug releases from the micelles were observed. These nano-micelles escaped the lysosomes and released doxorubicin and quercetin faster in the cytoplasm which resulted in synergistic anticancer activity against MCF-7 breast cancer cells [52].

Rafael de Oliveira Pedro et al. reported a simple method in which chitosan self-assembled amphiphilic nanoparticles loaded with quercetin were made for treating MCF-7 breast cancer cell lines. The results indicated $83 \%$ of quercetin entrapment in nanoparticles with a diameter ranging from 235 to $312 \mathrm{~nm}$. A larger release profile at $\mathrm{pH} 5$ was observed. MTT assay revealed inhibitory effects of quercetin in nanoparticle form due to greater uptake by the cells. Nanoparticles were hemocompatible, showing the emergence of a novel drug delivery system for cancer therapy [53].

Juan-Juan Ma et al. developed Zein-chitosan nanoparticles for loading quercetin (ZCPs-Q) to increase its solubility in water. Results revealed that (ZCPs-Q) increases the solubility of quercetin and stability in water. Furthermore, ZCPsQ showed increased antioxidant activity in HepG2 cells $(E C 50=31.18 \mu \mathrm{g} / \mathrm{mL})$ as compared to free quercetin's $(\mathrm{EC} 50=41.02 \mu \mathrm{g} / \mathrm{mL})$. Thus, these nanoparticles are suitable for loading nutraceuticals for enhancing cellular uptake with an increased antioxidant profile [54].

Jalil Rashedi et al. formulated chitosan and quercetin nanoparticles by inotropic gelation method for loading 5-FU. Anticancer effects were observed on colon cancer of Wistar rats. Firstly, the tumor was induced by using 1, 2-dimethylhydrazine (DMH) and dextran sulfate sodium (DSS). Rats were provided with NPs in the form of an enema. Results revealed high encapsulation efficiency of quercetin with an increased release profile up to $24 \mathrm{~h}$. A decrease in microvascular density and mitosis rate was noticed in all the treatment groups as compared to the control group, confirming the promising nature of NPs in site-specific colorectal cancer [55].

Wenhao Nan et al. reported the protective effects of quercetin against ultraviolet-B radiations to protect skin damage, cancer and photoaging along with inflammation. Due to low hydrophilicity and percutaneous absorption, quercetin use was limited in topical preparations. For this, chitosan nanoparticles were made using sodium tripolyphosphate and quercetin was entrapped in these nanoparticles. Results revealed remarkable skin penetration of quercetin with better stability and low cytotoxicity in HaCat cells. The nanoparticles enhanced effect of quercetin by inhibiting NF-kB/COX-2 signaling pathway. It was concluded that quercetin loaded on chitosan TPP nanoparticles can be used as topical preparation against ultraviolet-b radiations [56].

Virginia Tzankova et al. reported the hepatoprotective role of quercetin by encapsulating it in chitosan/alginate nanoparticles prepared by gelation method. Wistar 
rats were taken and paracetamol-induced liver injury was treated with these nanoparticles which showed significantly decreased levels of serum transaminases ALT and AST and restored gluthation levels. In vitro cell viability of HepG2 cells decreased with the treatment of encapsulated quercetin in nanoparticles. It was concluded that chitosan/alginate nanoparticles loaded with quercetin could be a better therapeutic option against oxidative stress-induced liver injuries [57].

Li Lv et al. reported a new therapeutic drug delivery system by incorporating doxorubicin and quercetin on biotin-decorated nanoparticles. The cytotoxic study was done on MCF-7 breast cancer cell lines and ADR. By inhibition of P-glycoprotein, enhanced cytotoxic effects were observed in biotin decorated nanoparticles as compared to nanoparticles without biotin. Thus it was concluded that MDR cancer cells can be treated with these novel nanoparticles [58].

\section{Conclusion}

This review examined that quercetin is a safe dietary supplement with a variety of biological functions in animals as well as in humans. Majority of the literature showed its safety profile in animals as an antimicrobial, antidiabetic, anticancer, antioxidant and anti-inflammatory agent. However, further evaluation in this regard with accurate outcomes is much needed. Poor solubility and oral bioavailability of quercetin were a major problem in its use which was managed by making its complexes with polymers and metal ions in sustained released microspheres, nanospheres and liposomal dosage forms. Synergistic effects of quercetin with anticancer, antimicrobials, antidiabetics and anti-inflammatory agents make it an interesting compound for exploring new treatment modalities for acute and chronic human diseases with lesser side effects and improved efficacy.

Acknowledgements The authors are very much thankful to the Department of Pharmaceutics, Faculty of pharmacy, Baha-Uddin-Zakariya university Multan for providing research facilities and Higher Education Commission (HEC) of Pakistan for providing the funding of the project.

\section{Declarations}

Conflict of interest The authors have indicated that they have no other conflicts of interest with regard to the content of this article.

\section{References}

1. Ozgen $\mathrm{S}$ et al (2016) Antioxidant activity of quercetin: a mechanistic review. Turk J Agric-Food Sci Technol 4(12):1134-1138

2. Xu D et al (2019) Antioxidant activities of quercetin and its complexes for medicinal application. Molecules 24(6):1123

3. Srimathi Priyanga K, Vijayalakshmi K (2017) Investigation of antioxidant potential of quercetin and hesperidin: an in vitro approach. Asian J Pharm Clin Res 10:83-86 
4. Farhadi $\mathrm{F}$ et al (2019) Antibacterial activity of flavonoids and their structure-activity relationship: an update review. Phytother Res 33(1):13-40

5. Mortale S, Karuppayil S (2018) Review on combinatorial approach for inhibiting Candida albicans biofilm. Am J Clin Microbiol Antimicrob 1:1-10

6. Al Aboody MS, Mickymaray SJA (2020) Anti-fungal efficacy and mechanisms of flavonoids. Antibiotics 9(2):45

7. Lalani S, Poh CLJV (2020) Flavonoids as antiviral agents for Enterovirus A71 (EV-A71). Viruses 12(2):184

8. Colunga Biancatelli RML et al (2020) Quercetin and vitamin C: an experimental, synergistic therapy for the prevention and treatment of SARS-CoV-2 related disease (COVID-19). Front Immunol 11:1451

9. Boudiaf F et al (2020) Anti-diabetic role of quercetin and cinnamon on neurobehavioral alterations and biochemical parameters of induced diabetics rats. J Anim Behav Biometeorol 8(3):190-195

10. Sharma G et al (2010) Anti-diabetic, anti-oxidant and anti-adipogenic potential of quercetin rich ethyl acetate fraction of Prunus persica. Pharmacognosy J 10(3)

11. Hisaka T et al (2020) Quercetin suppresses proliferation of liver cancer cell lines in vitro. Anticancer Res 40(8):4695-4700

12. Zhou $\mathbf{J}$ et al (2017) Investigation of the anti-cancer effect of quercetin on HepG2 cells in vivo. PLoS ONE 12(3):e0172838

13. Guan F et al (2021) Anti-rheumatic effect of quercetin and recent developments in nano formulation. RSC Adv 11(13):7280-7293

14. Shastrala K et al (2021) Synthesis, characterization, and pharmacological evaluation of some metal complexes of quercetin as P-gp inhibitors. Future J Pharm Sci 7(1):1-13

15. Nathiya S., Durga M, Devasena TJA (2014) Quercetin, encapsulated quercetin and its applicationa review. Analgesia. 10(11)

16. Batiha GES et al (2020) The pharmacological activity, biochemical properties, and pharmacokinetics of the major natural polyphenolic flavonoid: quercetin. Foods 9(3):374

17. Li Y et al (2016) Quercetin, inflammation and immunity. Nutrients 8(3):167

18. Wang J et al (2018) Quercetin increases the antioxidant capacity of the ovary in menopausal rats and in ovarian granulosa cell culture in vitro. J Ovarian Res 11(1):1-11

19. Suematsu, N., M. Hosoda, and K.J.N.1. Fujimori, Protective effects of quercetin against hydrogen peroxide-induced apoptosis in human neuronal SH-SY5Y cells. 2011. 504(3): p. 223-227.

20. Lesjak M et al (2018) Antioxidant and anti-inflammatory activities of quercetin and its derivatives. J Funct Foods 40:68-75

21. Zheng Y-Z et al (2017) Antioxidant activity of quercetin and its glucosides from propolis: a theoretical study. Sci Rep 7(1):1-11

22. Pahal V, Devi U, Dadhich KS, Quercetin, a secondary metabolite present in methanolic extract of Calendula officinalis, is a potent inhibitor of peptide deformylase, undecaprenyl pyrophosphate synthase and DNA primase enzymes of Staphylococcus aureus: an in vitro and in silico result analysis. pp 216-225

23. da Costa Júnior SD et al (2018) Antibacterial and antibiofilm activities of quercetin against clinical isolates of Staphyloccocus aureus and Staphylococcus saprophyticus with resistance profile. Int J Environ Agric Biotechnol 3(5):266213

24. Adamczak A, Ożarowski M, Karpiński TMJJOCM (2020) Antibacterial activity of some flavonoids and organic acids widely distributed in plants. J Clin Med 9(1):109

25. Li F et al (2018) The simultaneous loading of catechin and quercetin on chitosan-based nanoparticles as effective antioxidant and antibacterial agent. Food Res Int 111:351-360

26. Sarangapani S, Jayachitra AJFFIH (2018) Targeting biofilm inhibition using quercetin-interaction with bacterial cell membrane and ROS mediated biofilm control. Funct Foods Health Dis 8(6):292-306

27. Gao M et al (2016) Quercetin assists fluconazole to inhibit biofilm formations of fluconazole-resistant Candida albicans in in vitro and in vivo antifungal managements of vulvovaginal candidiasis. Cell Physiol Biochem 40(3-4):727-742

28. Rocha MFG et al (2019) Antifungal effects of the flavonoids kaempferol and quercetin: a possible alternative for the control of fungal biofilms. Biofouling 35(3):320-328

29. Shishodia SK et al (2020) SEM and qRT-PCR revealed quercetin inhibits morphogenesis of Aspergillus flavus conidia via modulating calcineurin-Crz1 signalling pathway. Mycology 11(2):118-125 
30. Oliveira V et al (2016) Quercetin and rutin as potential agents antifungal against Cryptococcus spp. Br J Biol 76:1029-1034

31. Milanezi FG et al (2019) Antioxidant, antimicrobial and cytotoxic activities of gold nanoparticles capped with quercetin. Saudi Pharm J 27(7):968-974

32. Dewi B, et al (2020) Effectivity of quercetin as antiviral to dengue virus-2 strain New Guinea $\mathrm{C}$ in Huh 7-it 1 cell line. In: IOP conference series: earth and environmental science. IOP Publishing

33. Rojas Á et al (2016) Effect of quercetin on hepatitis C virus life cycle: from viral to host targets. Sci Rep 6(1):1-9

34. Wong $\mathrm{G}$ et al (2017) Antiviral activity of quercetin-3- $\beta$-OD-glucoside against Zika virus infection. Virol Sin 32(6):545-547

35. Di Pierro $F$ et al (2021) Potential clinical benefits of quercetin in the early stage of COVID-19: results of a second. Pilot Randomized Control Open-Label Clin Trial 14:2807

36. Wu W et al (2016) Quercetin as an antiviral agent inhibits influenza A virus (IAV) entry. Viruses $8(1): 6$

37. Shaikhomar OA, Bahattab OSJSJBR (2021) Physiological Effect of Quercetin as a Natural Flavonoid to be used as Hypoglycemic Agent in Diabetes Mellitus Type II Rats. Saudi J Biomed Res 6(1):10-17

38. Hemmati M, et al. (2018) Protective effects of quercetin on hyperglycemia and stress proteins expression in rats with streptozocin-induced diabetes. Modern Care J. 15(2)

39. Singh $\mathbf{J}$ et al (2018) Design, optimization, characterization and in-vivo evaluation of Quercetin enveloped Soluplus $₫ / P 407$ micelles in diabetes treatment. Artif Cells Nanomed Biotechnol 46(3):S546-S555

40. Abu Ayana MA et al (2017) Efficacy of quercetin on alveolar bone structure of rats with induced diabetes. Alex Dent J 42(2):141-146

41. Lei C-S et al (2018) Effects of quercetin combined with anticancer drugs on metastasis-associated factors of gastric cancer cells: in vitro and in vivo studies. J Nutr Biochem 51:105-113

42. Liu $\mathrm{H}$ et al (2019) Effect of quercetin on the anti-tumor activity of cisplatin in EMT6 breast tumorbearing mice. Obstet Gynecol Sci 62(4):242-248

43. Xu G et al (2018) Enhancing the anti-ovarian cancer activity of quercetin using a self-assembling micelle and thermosensitive hydrogel drug delivery system. RSC Adv 8(38):21229-21242

44. Shen Y, TanTai JJDD (2020) Development, and therapy, co-delivery anticancer drug nanoparticles for synergistic therapy against lung Cancer Cells. Drug Des Dev Therapy 14:4503

45. Karliana D, Anwar E, Bahtiar AJIJOAP (2019) Formulation and evaluation of quercetin nanoparticle gel for osteoarthritis. Int J Appl Pharm 11(5):54-59

46. Lee HN et al (2018) Anti-inflammatory effect of quercetin and galangin in LPS-stimulated RAW264. 7 macrophages and DNCB-induced atopic dermatitis animal models. Int J Mol Med 41(2):888-898

47. Saeedi-Boroujeni A, Mahmoudian-Sani M-RJJOI (2021) Anti-inflammatory potential of Quercetin in COVID-19 treatment. J Inflamm 18(1):1-9

48. Ozyel B et al (2021) Anti-inflammatory effects of quercetin on high-glucose and pro-inflammatory cytokine challenged vascular endothelial cell metabolism. Mol Nutr Food Res 65(6):2000777

49. Patra $\mathrm{M}$ et al (2017) Calcium phosphate-quercetin nanocomposite (CPQN): A multi-functional nanoparticle having $\mathrm{pH}$ indicating, highly fluorescent and anti-oxidant properties. Coll Surf B Biointerfaces 154:63-73

50. Hassanien MM, Saad EA, Radwan KHJJOAPS (2020) Antidiabetic activity of cobalt-quercetin complex: A new potential candidate for diabetes treatment. J Appl Pharm Sci 10(12):044-052

51. Refat MS et al (2021) Quercetin/Zinc complex and stem cells: a new drug therapy to ameliorate glycometabolic control and pulmonary dysfunction in diabetes mellitus: Structural characterization and genetic studies. PLoS ONE 16(3):e0246265

52. $\mathrm{Mu} \mathrm{Y}$ et al (2019) pH-sensitive amphiphilic chitosan-quercetin conjugate for intracellular delivery of doxorubicin enhancement. Carbohydr Polym 223:115072

53. de Oliveira Pedro R et al (2018) Self-assembled amphiphilic chitosan nanoparticles for quercetin delivery to breast cancer cells. Eur J Pharm Biopharm 131:203-210

54. Ma J-J et al (2018) Cellular uptake and intracellular antioxidant activity of zein/chitosan nanoparticles incorporated with quercetin. J Agric Food Chem 66(48):12783-12793

55. Rashedi J et al (2019) Anti-tumor effect of quercetin loaded chitosan nanoparticles on induced colon cancer in wistar rats. Adv Pharm Bull 9(3):409 
56. Nan W et al (2018) Topical use of quercetin-loaded chitosan nanoparticles against ultraviolet B radiation. Front Pharmacol 9:826

57. Tzankova V et al (2017) Hepatoprotective and antioxidant activity of quercetin loaded chitosan/ alginate particles in vitro and in vivo in a model of paracetamol-induced toxicity. Biomed Pharm 92:569-579

58. Lv L et al (2016) Quercetin and doxorubicin co-encapsulated biotin receptor-targeting nanoparticles for minimizing drug resistance in breast cancer. Oncotarget 7(22):32184

Publisher's Note Springer Nature remains neutral with regard to jurisdictional claims in published maps and institutional affiliations. 